\title{
Piezoelectric Control of the Exciton Wave Function in Colloidal CdSe/ CdS Nanocrystals
}

\author{
Carlos Segarra, ${ }^{\dagger}$ Juan I. Climente, ${ }^{\dagger}$ Anatolii Polovitsyn, ${ }^{\ddagger}$ Fernando Rajadell, ${ }^{\dagger}$ Iwan Moreels, ${ }^{\dagger}$ \\ and Josep Planelles ${ }^{*} \dagger$
}

${ }^{\dagger}$ Departament de Química Física i Analítica, Universitat Jaume I, E-12080 Castelló de la Plana, Spain
${ }^{\ddagger}$ Istituto Italiano di Tecnologia, Via Morego 30, IT-16163 Genova, Italy

Supporting Information

ABSTRACT: Using multiband $k \cdot p$ calculations, we show that strain-engineered piezoelectricity is a powerful tool to modulate the electron-hole spatial separation in a wide class of wurtzite CdSe/CdS nanocrystals. The inherent anisotropy of the hexagonal crystal structure leads to anisotropic strain and, consequently, to a pronounced piezoelectric field along the $c$ axis, which can be amplified or quenched through a proper design of the core-shell structure. The use of large cores and thick shells promotes a gradual departure from quantum confined nanocrystals to a regime dominated by piezoelectric confinement. This allows excitons to evolve from the usual type-I and quasi-type-II behavior to a type-II behavior in dot-in-dots, dot-in-rods, rod-in-rods, and dot-in-plates. Piezoelectric fields explain experimental observations for giant-shell nanocrystals, whose time-resolved photoluminescence reveals long exciton lifetimes for large cores, contrary to the expectations of standard quantum confinement models. They also explain the large differences in exciton lifetimes reported for

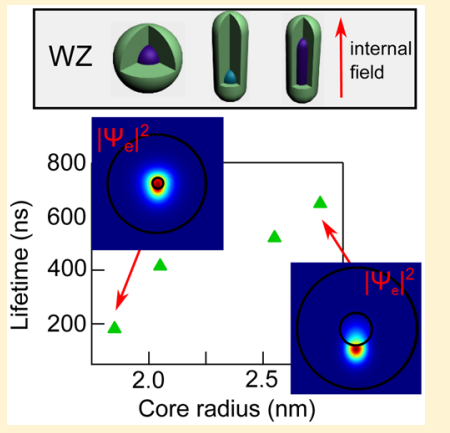
different classes of $\mathrm{CdSe} / \mathrm{CdS}$ nanocrystals.

$\mathrm{T}$ he spatial separation between electrons and holes in colloidal semiconductor nanocrystals (NCs) is a critical parameter that impacts the exciton emission lifetime, ${ }^{1}$ Auger recombination rate, ${ }^{2-4}$ electron-hole exchange interaction, ${ }^{5}$ charge separation time, ${ }^{6}$ and other properties of interest for optoelectronic devices such as LEDs, lasers, photovoltaic cells, or photocatalysts. ${ }^{7}$ Control of the electron-hole separation is usually achieved by means of band gap engineering in core/ shell hetero-NCs. An additional control mechanism was proposed for $\mathrm{CdTe} / \mathrm{ZnSe} \mathrm{NCs}$, which exploited the epitaxial strain arising from the large lattice mismatch between the two materials $(13.4 \%){ }^{8}$ The growth of a thick compressive shell around the core shifts the core band edges via the deformation potential, leading to a gradual transition from (unstrained) type-I band alignment to a (fully strained) type-II one. Subsequently, the influence of strain on the band structure and electron-hole wave functions was investigated in other core/shell structures including $\mathrm{CdSe} / \mathrm{CdTe}(6.7 \%$ lattice mismatch), ${ }^{6,9} \mathrm{ZnSe} / \mathrm{ZnTe}(7 \%),{ }^{10,11}$ and $\mathrm{CdS} / \mathrm{ZnS}(7 \%) .^{12-14}$

In materials with a smaller lattice mismatch, strain-driven localization of carriers is less efficient, as the band edges are shifted only moderately. This is unfortunate because weakly strained NCs are less prone to interfacial defects and, hence, are preferred for their higher photoluminescence quantum yields. ${ }^{15}$ This is the case of CdSe/CdS NCs, ${ }^{16}$ (4.4\% for zinc-blende (ZB), $4 \%$ and $3.8 \%$ along the $a$ and $c$ axes in wurtzite (WZ)), which are structures of particular interest owing to their monodispersity, reduced blinking, narrow emission line width and high quantum yield. ${ }^{17,18}$ Several types of core-shell CdSe/ $\mathrm{CdS}$ heterostructures have been synthesized in the past decade: dot-in-dots (DiDs), ${ }^{2,15,17-21}$ dot-in-rods (DiRs), ${ }^{22-25}$ dot-inplates (DiPs), ${ }^{26}$ rod-in-rods (RiRs), ${ }^{27,28}$ tetrapods, ${ }^{29,30}$ and octapods. ${ }^{31}$ Carrier localization in these systems is generally assumed to be set by quantum confinement. The smaller gap of CdSe favors localization of both electron and hole inside the core (type-I exciton), but as the core size decreases, the electron kinetic energy allows it to overcome the $\mathrm{CB}$ offset barrier, delocalizing over both core and shell (quasi-type-II exciton). 2,5,24,32 Note, however, that the spatial separation between electron and hole is typically restricted to small core systems and is ultimately limited by Coulomb interaction, which binds the electron to the vicinity of the hole. ${ }^{32,33}$

Very recently, a study on $\mathrm{CdSe} / \mathrm{CdS}$ RiRs with giant core and shell reported extremely long exciton lifetimes (up to 4400 $n s){ }^{28}$ This is 1 order of magnitude longer than any reported values for giant-shell $\mathrm{CdSe} / \mathrm{CdS} \mathrm{DiDs}{ }^{5,18}$ and two longer than those of DiR or core-only NCs, ${ }^{24,28}$ which reflects a truly typeII behavior with well separated electrons and holes. It was shown that such exotic properties followed from the straininduced piezoelectric (PZ) charges arising at the $\mathrm{CdSe} / \mathrm{CdS}$ interfaces along the $\mathrm{WZ} c$ axis. Strain-induced $\mathrm{PZ}$ fields have been shown to be important in several epitaxial structures, including $\mathrm{CdSe} / \mathrm{CdS}$ superlattices and $\mathrm{III}-\mathrm{V}$ quantum dots. $^{34-36}$ This raises the question of how disruptive they can be in colloidal structures, if they are only important in large RiRs due to the anisotropic shape and weak longitudinal

Received: March 18, 2016

Accepted: May 25, 2016

Published: May 25, 2016 


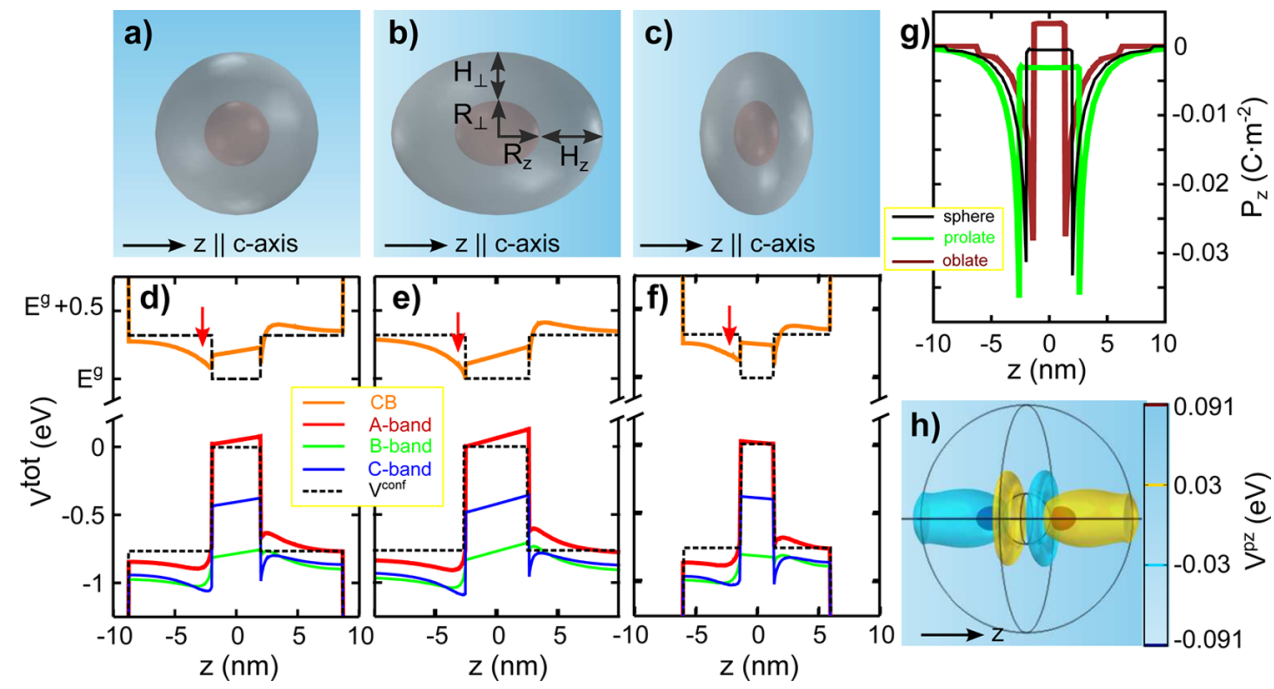

Figure 1. (a) -(c) schematic of spherical, prolate, and oblate DiDs. (d) $-(\mathrm{f}) \mathrm{CB}$ and $\mathrm{VB}$ confining and total potential for a spherical $\mathrm{DiD}$ with core radius $R=2 \mathrm{~nm}$ and shell thickness $H=7 \mathrm{~nm}(\mathrm{~d})$, prolate $\mathrm{DiD}$ with aspect ratio 1.3:1 $\left(R_{z}=1.3 R_{\perp}\right.$ and $\left.H_{z}=1.3 H_{\perp}\right)(\mathrm{e})$ and oblate DiD with aspect ratio 0.7:1 (f). (g) strain-induced polarization along the $c$-axis for the three kinds of $\mathrm{DiD}$. (h) isosurface representation of the piezoelectric potential for the spherical DiD. In (d)-(f), $E^{g}$ is the bulk band gap.

confinement or if it is possible to use them as an efficient charge separation mechanism in other kinds of WZ NCs, with smaller core dimensions and different shapes. Potential signatures of $\mathrm{PZ}$ fields have been observed in $\mathrm{CdSe} / \mathrm{CdS}$ $\mathrm{DiDs}^{37,38}$ and DiRs, ${ }^{37}$ but it has been mostly overlooked because calculations for a few particular structures deemed it a minor effect. ${ }^{16,37,39}$ In this Letter, we show that with appropiate structural design, piezoelectricity indeed becomes a major factor determining the electron-hole separation in most kinds of WZ CdSe/CdS NCs.

We consider excitons in $\mathrm{CdSe} / \mathrm{CdS}$ NCs where both core and shell present WZ structure. A few theoretical considerations are useful for the discussion of the results. The excitonic electron and hole Hamiltonians read

$$
H_{j}=H_{j}^{\mathrm{kin}}+V_{j}^{\mathrm{conf}}+V_{j}^{\mathrm{str}}+V_{j}^{\mathrm{pz}}+V_{j}^{\mathrm{e}-\mathrm{h}}
$$

where $j=e$ or $h$ stands for electron or hole, $H_{j}^{\text {kin }}$ is the kinetic energy term, $V_{j}^{\text {conf }}$ the confining potential defined by the band offsets between bulk CdSe and CdS, $V_{j}^{\text {str }}$ the strain induced deformation potential, $V_{j}^{\mathrm{pz}}$ the strain induced $\mathrm{PZ}$ potential and $V_{j}^{\mathrm{e}-\mathrm{h}}$ is Coulomb attraction exerted upon carrier $j$ by the other carrier. Special attention will be paid to the PZ potential term. In WZ, strain shifts the atomic nuclei inducing a PZ polarization vector

$$
\mathbf{P}=\left(\begin{array}{c}
e_{15} \epsilon_{x z} \\
e_{15} \epsilon_{y z} \\
e_{31}\left(\epsilon_{x x}+\epsilon_{y y}\right)+e_{33} \epsilon_{z z}
\end{array}\right)
$$

where $e_{r s}$ are $\mathrm{PZ}$ coefficients and $\epsilon_{i j}$ strain tensor components. Notice that the polarization along the $c$-axis, $P_{z}$, is particularly important because it involves diagonal strain components, which are larger than the off-diagonal (shear) ones. Because $e_{33}$ $\approx-2 e_{31}$, sizable polarization $P_{z}$ is expected when strain is anisotropic, that is, $\left(\epsilon_{x x}+\epsilon_{y y}\right) / 2 \neq \epsilon_{z z}$. The polarization is different in core and shell materials, as they experience different strain forces and have different piezoelectric coefficients. As a consequence, $\mathrm{PZ}$ charges $\rho(\mathbf{r})=-\nabla \mathbf{P}$ arise near the interface. Again, these are especially important along the $c$ axis. The PZ charges give rise to a $\mathrm{PZ}$ field according to the Poisson equation, $\nabla \epsilon(\mathbf{r}) \nabla \phi^{\mathrm{pz}}(\mathbf{r})=-4 \pi \rho(\mathbf{r})$, and the PZ potential is finally obtained as $V_{j}^{\mathrm{pz}}= \pm q \phi^{\mathrm{pz}}$, where plus and minus sign apply to $j=e$ and $j=h$, respectively, and $q$ is the electron charge.

We start by investigating spheroidal $\mathrm{DiDs}$ like those illustrated in Figure $1 \mathrm{a}-\mathrm{c}$. Consider first a fully spherical DiD. Figure $1(\mathrm{~d})$ shows the $\mathrm{CB}$ and $\mathrm{VB}$ potential profiles. Dashed lines represent the confinement potential $V^{\text {conf }}$, whereas solid lines represent the total single-particle potential, including strain-induced deformation potential and PZ terms, $V^{\text {tot }}=V^{\text {conf }}$ $+V^{\text {str }}+V^{\mathrm{pz}}$. By inspecting the CB potential, orange line, one can see that the inclusion of $V^{\text {str }}+V^{\mathrm{pr}^{\mathrm{z}}}$ has three important effects. First, the core potential is shallower. This is a consequence of $V^{\text {str }}$ (see Figure $\mathrm{S} 1$ in the Supporting Information (SI) or ref 16). Second, the core bottom develops a built-in PZ field of $15 \mathrm{mV} / \mathrm{nm}$. This is a consequence of the PZ term, $V^{\mathrm{pz}}$ (see Figure S1 in SI or ref 16). Third, and most important, because the $\mathrm{CB}$ potential is shallow, the positive $\mathrm{PZ}$ charges accumulating at the bottom $\mathrm{CdSe} / \mathrm{CdS}$ interface form a potential well in the shell where electrons can be trapped (red arrow in Figure 1d).

For holes the situation is different. We have three subbands: A-, B- and C-band. All three subbands are shifted upward by $V^{\text {str }}$. Contrary to electrons, this now results in a slightly deeper confinement. The effect of $V^{\mathrm{pz}}$ in the core is the same as for electrons, but owing to the different charge sign, holes will be pushed to the upper $\mathrm{CdSe} / \mathrm{CdS}$ interface, thus favoring electron-hole separation. On the other hand, because the Aband confinement potential $V^{\text {conf }}$ is very deep (see dashed lines), the negative $\mathrm{PZ}$ charges accumulating at the top CdSe/ $\mathrm{CdS}$ interface do not suffice to localize the hole ground state outside the core.

Considering a prolate $\mathrm{DiD}$ instead of spherical, the PZ field increases (up to $23 \mathrm{mV} / \mathrm{nm}$ in Figure 1e). Conversely, for an oblate $\mathrm{DiD}$ the field is reduced and eventually the sign is even reversed, see Figure 1f.

The presence of a significant PZ field in the spherically symmetric $\mathrm{DiD}$ implies that even for structures with isotropic confinement, the inherent anisotropy of the WZ lattice leads 


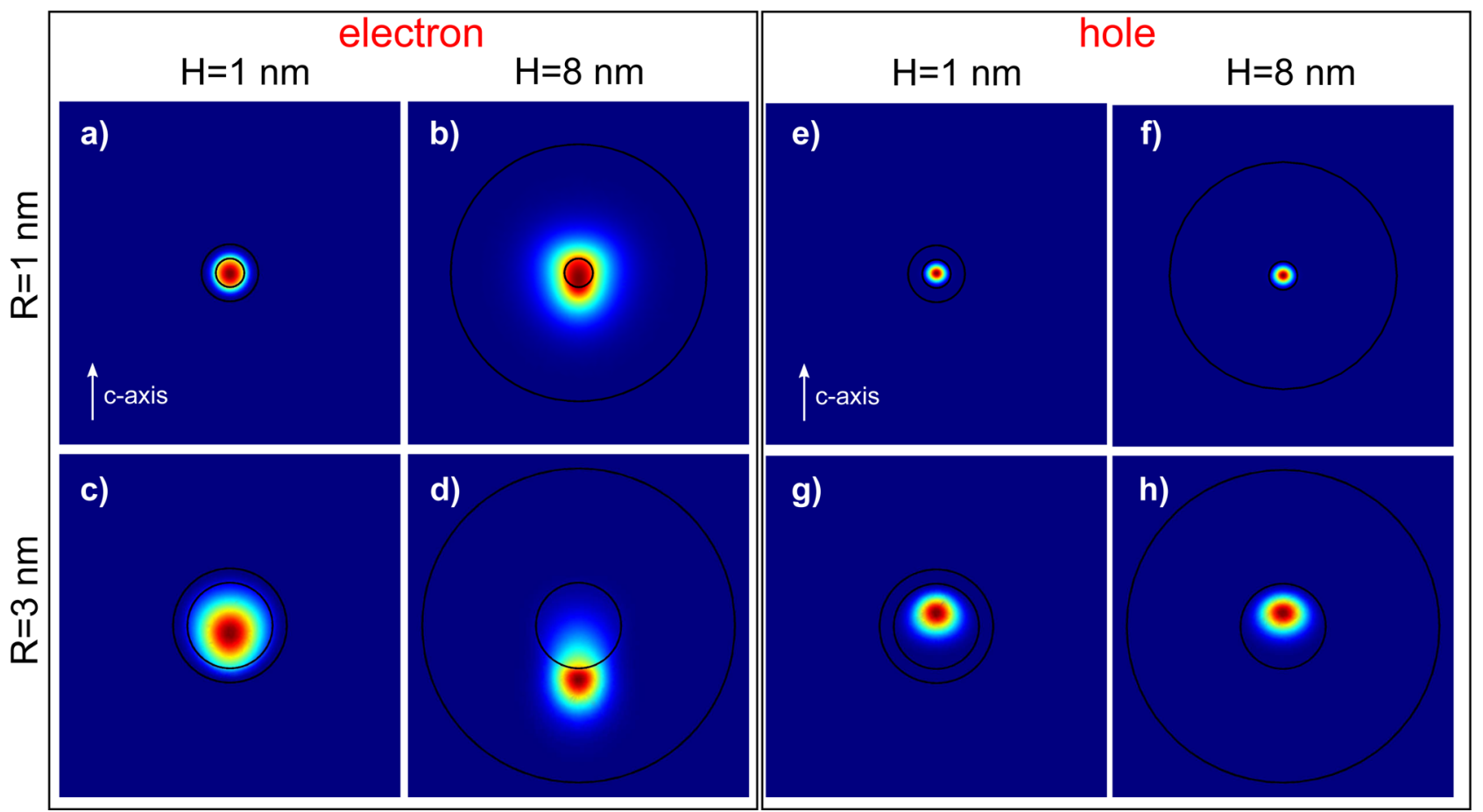

Figure 2. (a)-(d) Excitonic electron charge density in spherical DiDs with different core radius $R$ and shell thickness $H$. (e-h) Same for excitonic hole. The electron moves into the shell for large cores and thick shells, panel $\mathrm{d}$.

pronounced polarization near the $\mathrm{CdSe} / \mathrm{CdS}$ interface, see black line in Figure $1 \mathrm{~g}$ and notice the different weight of $\epsilon_{z z}$ and $\epsilon_{\perp}=\left(\epsilon_{x x}+\epsilon_{y y}\right) / 2$ in Figure S2 of the SI. This leads to a mostly dipolar PZ potential, as shown in Figure $1 \mathrm{~h}$. The strain anisotropy can be conveniently manipulated by using anisotropic geometries. Prolate structures favor $\left|\epsilon_{z z}\right|>\left|\epsilon_{\perp}\right|$ in the core, whereas oblate ones do the opposite, see Figure S2 in SI. This translates into more negative (positive) $P_{z}$ values, see green (brown) line in Figure 1g. We then conclude that the shape of core/shell structures can be used to enhance, cancel or reverse PZ fields.

The next question is whether the magnitude of the attainable PZ fields is enough to influence excitonic wave functions in realistic DiDs. In Figure 2, we compare the exciton electron $(\mathrm{a}-\mathrm{d})$ and hole $(\mathrm{e}-\mathrm{h})$ charge densities for spherical DiDs with different dimensions. For small cores and thin shells $(R=1 \mathrm{~nm}$, $H=1 \mathrm{~nm}$ ), both electron (Figure 2a) and hole (Figure 2e) are centered in the core. Growing a giant shell $(H=8 \mathrm{~nm})$ instead increases the core compression. As a result, $V^{\text {str }}$ reduces the $\mathrm{CB}$ confinement barrier (recall Figure 1d) and the electron wave function starts leaking into the shell in spite of the Coulomb attraction, Figure $2 \mathrm{~b}$. The hole, by contrast, remains in the core because of the high confinement barrier, Figure $2 \mathrm{f}$.

If the core is large enough $(R=3 \mathrm{~nm})$ but the shell is thin $(H$ $=1 \mathrm{~nm}$ ), electron and hole are pushed toward opposite sides of the core along the $c$-axis by the PZ field, Figure $2 \mathrm{c}, \mathrm{g}$, but the resulting overlap is still substantial. The most remarkable effect of piezoelectricity takes place when a large core is surrounded by a giant shell. As can be seen in Figure $2 \mathrm{~d}$, in this case the electron escapes from the core and localizes in the shell near the CdSe/CdS interface. The electron is actually taking advantage of the potential minimum originated in the positive $\mathrm{PZ}$ interface charge, red arrow in Figure 1d. Because the hole remains in the core, Figure $2 \mathrm{~h}$, the spatial separation between the two carriers becomes large. In other words, by using large
$\mathrm{CdSe}$ cores and growing giant $\mathrm{CdS}$ shells around, a gradual transition from type-I to type-II exciton driven by $\mathrm{PZ}$ is feasible.

For a comprehensive view of the effect of $\mathrm{PZ}$ on the exciton wave function of $\mathrm{DiDs}$, we compute the electron-hole overlap integral squared, $S_{\mathrm{eh}}^{2}=\left\langle\Psi_{\mathrm{e}} \mid \Psi_{\mathrm{h}}\right\rangle^{2}$, which is directly proportional to the radiative exciton decay rate. ${ }^{1}$ In Figure 3, we compare the overlap (a) excluding and (b) including strain and PZ effects $\left(V^{\text {str }}\right.$ and $\left.V^{\mathrm{pz}}\right)$. The first case corresponds to DiDs governed by quantum confinement and Coulomb interactions only, which is the scenario assumed so far in the literature. The results are

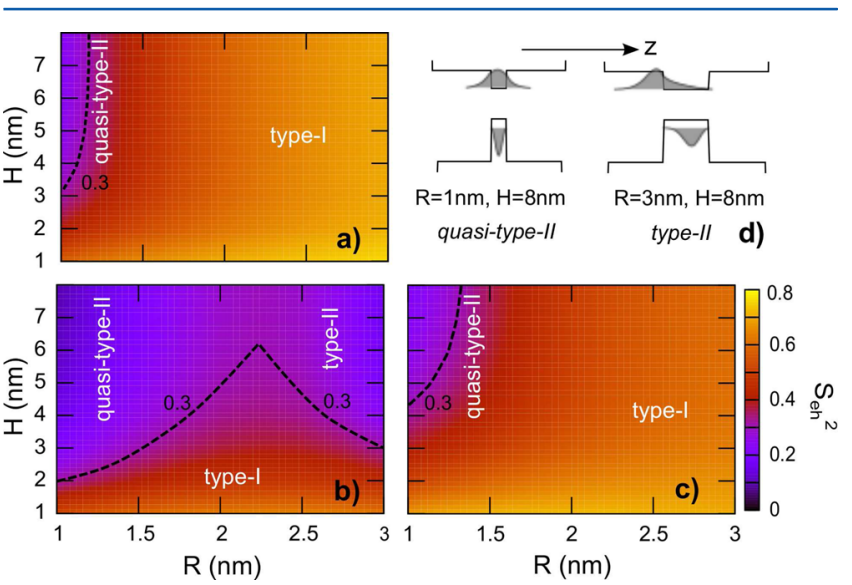

Figure 3. Electron-hole overlap squared for excitons confined in spherical WZ DiDs without (a) and with (b) strain and piezoelectric effects. The inclusion of strain and PZ reduces overlaps (compare the (dashed) isoline for $S_{e h}^{2}=0.3$ in the two panels) and introduces a typeII regime for large cores and thick shells. (c) Electron (top) and hole (bottom) charge densities along the $c$ axis for two DiDs of panel (b), evidencing quasi-type-II $(R=1 \mathrm{~nm})$ and type-II $(R=3 \mathrm{~nm})$ exciton character. (d) Same as (a) and (b) but for strained ZB DiDs. The absence of $\mathrm{PZ}$ in this case translates into absence of type-II regime. 
essentially the same as described, for example, in Figure 1f of ref 2. For core radii $R=1-1.5 \mathrm{~nm}$, increasing the shell thickness $H$ leads into a quasi-type-II regime, where the electron leaks into the shell due to the high kinetic energy in the core. For larger $R$, the electron stays inside the core no matter how thick the shell, and a type-I exciton is obtained with strong overlap values, similar to core-only samples.

The behavior changes drastically when strain and PZ effects are taken into account, Figure $3 \mathrm{~b}$. For thick shells, the quasitype-II regime extends toward larger core radii $(R \lesssim 2 \mathrm{~nm})$. This is a consequence of strain making $C B$ confinement shallower (see also $S_{\text {eh }}^{2}$ for $V^{\text {str }} \neq 0$ and $V^{\mathrm{pz}}=0$ in Figure.S4 of SI). Moreover, with further increasing core radius $(R \gtrsim 2.5$ $\mathrm{nm}$ ), instead of retrieving a type-I behavior the overlap decreases again. This is a consequence of the formation of $\mathrm{PZ}$ induced type-II excitons, as shown in Figure 2d,h. We stress that the nature of the reduced $S_{\text {eh }}$ values is different on both sides of the figure. For small $R$ (quasi-type-II regime), it is driven by the strong core confinement. For large $R$ (type-II regime), it is driven by strain-induced PZ. The different localization of electron and hole charge densities in each case is clearly seen in Figure 3c. For comparison, in Figure 3d we also represent $S_{\text {eh }}^{2}$ for ZB core/ZB shell CdSe/CdS DiDs. Strain is still present in such structures, but the dipolar PZ potential is quenched because of the cubic lattice symmetry. The resulting behavior is similar to that of unstrained WZ NCs, Figure 3a, and it is qualitatively different from that of realistic WZ DiDs, Figure $3 \mathrm{~b}$. We shall see below that this difference between WZ and $\mathrm{ZB}$ DiDs is fully consistent with experimental data.

The PZ control of the electron-hole overlap of WZ DiDs we report in Figure 3b, which should have important consequences on exciton lifetimes, electron-hole exchange integrals, and so forth, is robust against deviations from sphericity (Figure S5 in SI). Since the actual value of the CB offset is often discussed, ${ }^{32}$ we have also studied the effect of changing from $0.32 \mathrm{eV}$ (the value used in Figure 3) to a lower estimate of $0.20 \mathrm{eV}$. The results are qualitatively unchanged, but the overlap values in the type-II regime become manifestly lower than those in the quasitype-II one, see Figure S6 in SI. It is also worth noting that although the PZ field is very efficient in separating carriers, its influence on the energy is modest. The exciton energy with and without strain differs at most in few tens of millielectronvolts (see Figure S7 in SI and ref 16).

To test the above predictions, we synthesized two series of giant-shell $\mathrm{WZ} \mathrm{DiDs}$ with variable $\mathrm{CdSe}$ core radii and $\mathrm{CdS}$ shell thickness of about 20 and $15 \mathrm{ML}$, respectively (Figure 4a, see table S2 in SI for structural and optical properties). The resulting PL peak position varies between 648 and $663 \mathrm{~nm}$ and the time-resolved PL traces show a nonexponential decay (see Figure $4 \mathrm{~b}, \mathrm{c}$ for the $20 \mathrm{ML}$ shell thickness series or Figure S10 in SI for the $15 \mathrm{ML}$ one). Figure $4 \mathrm{c}$ shows that as the core size increases, we observe a slower decay. Note however that previous measurements ${ }^{18}$ suggested the opposite, and the series with $15 \mathrm{ML}$ shell thickness does not reveal a clear trend; hence, further work remains needed to firmly establish this behavior. Corresponding lifetimes for both 20 and $15 \mathrm{ML}$ shell DiDs are plotted in Figure 4d using solid and open symbols, respectively. The values are calculated either from the time when the PL signal has decayed to $1 / e$ (dots) or $1 / e^{2}$ (triangles) of its initial value, or from a multiexponential fit (diamonds) to the decay trace (see SI for the analysis and summary of all components). Regardless of the core size dependence, the long lifetimes, especially for the large-core samples, are in clear contrast to
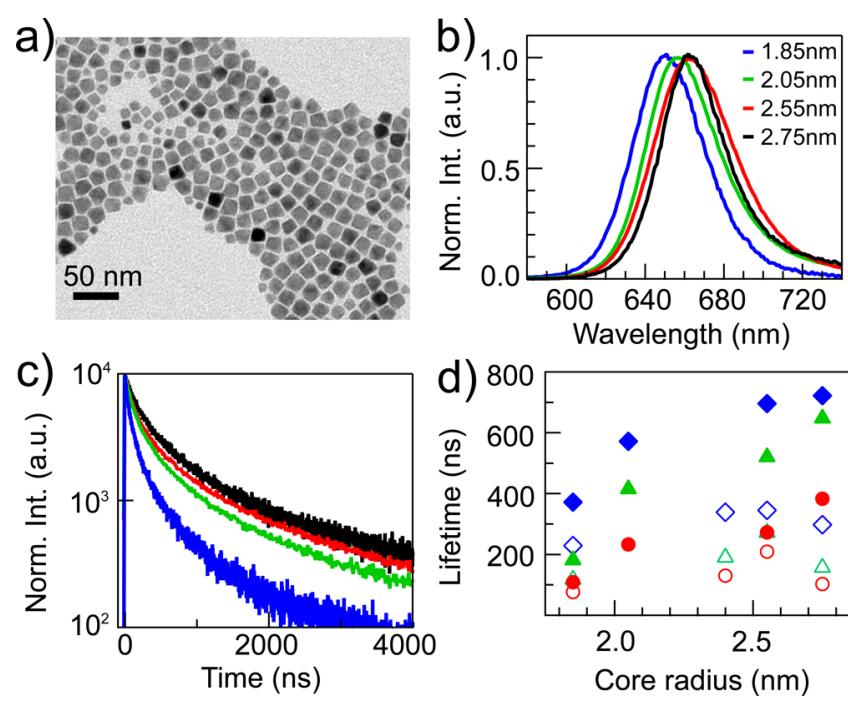

Figure 4. (a) Typical transmission electron microscope image of giantshell DiDs (core radius $2.05 \mathrm{~nm}, 20 \mathrm{ML}$ shell). (b) PL spectra for four different DiDs with $20 \mathrm{ML}$ shell. (c) Corresponding PL decay traces. (d) Resulting effective lifetimes determined from the $1 / e$ decay time (dots), $1 / e^{2}$ decay time (triangles), and from a fit to the decay traces using a multiexponential function (diamonds, see SI for details). Solid (open) symbols are used for the $20 \mathrm{ML}$ (15 ML) shell thickness series. The thinner shell gives shorter lifetimes. In general, lifetimes largely exceed those of giant-shell $\mathrm{ZB}$ DiDs.

expectations from the usual confinement picture for $\mathrm{CdSe} /$ $\mathrm{CdS}$, where electron and hole states are expected to become localized into the core, approaching lifetimes of core-only CdSe NCs (ca. 15-20 ns). Such long lifetimes are indicative of a type-II regime, and they support the theory of PZ fields as an efficient mechanism of charge separation. Our values also exceed measurements of fluorescence lifetimes in $\mathrm{ZB} \mathrm{CdSe/}$ CdS DiDs with moderately large cores $(R \approx 1.5 \mathrm{~nm})$ and thick shells $(H \approx 5.5 \mathrm{~nm})$ which have yielded values of about $30 \mathrm{~ns}^{21}$ This is again consistent with the theoretical predictions of Figure 3, which showed that PZ in thick-shell WZ DiDs leads to reduced electron-hole overlap as compared to $\mathrm{ZB}$ ones. Moreover, the strong differences in PL lifetime between the two samples series studied here highlight the sensitivity of the final lifetime to the shell thickness, even when it is grown to a regime where strong quantum confinement no longer influences the optical properties. This provides extra confirmation on PZ playing a significant role in giant-shell NCs with WZ crystal structure.

Having confirmed the influence of $\mathrm{PZ}$ fields in $\mathrm{DiDs}$, we next probe other WZ CdSe/CdS structures such as DiRs, DiPs and RiRs, which we model as ellipsoids with different degrees of anisotropy and different orientation of the $c$ axis. Let us consider first DiRs. Experiments available in the literature are generally consistent with a quantum confinement model, with a type-I band alignment and conduction band offsets between 0.1 and $0.3 \mathrm{eV}$. With decreasing core size, the excitons change from type-I to quasi-type-II behavior, ${ }^{24,32}$ yielding room temperature lifetimes between 8 and $40 \mathrm{~ns}$. $^{24,25,40}$ The absence of apparent PZ effects can be understood from two factors. First, typical cores for DiRs are small $(R \lesssim 2 \mathrm{~nm})$. The PZ dipole moment is then weak and, as noticed above (Figure 2 and Figure 3 ), $V^{p z}$ has a minor influence. Second, the shell surrounding the core is thin on the lateral sides (usually $1 \mathrm{~nm}$ or less). As compared to the giant shells of DiDs, the thin lateral shell of DiRs allows the 

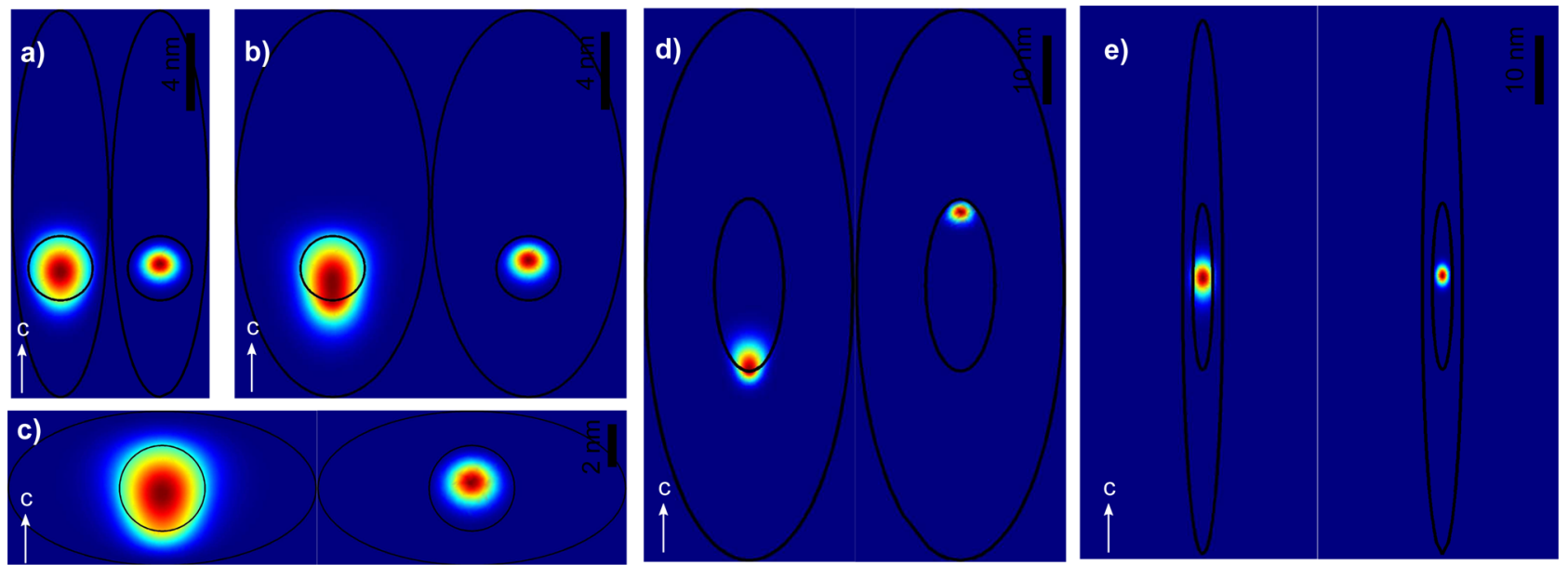

Figure 5. Excitonic charge density in different types of CdSe/CdS NCs. In each structure, the left (right) panel shows the electron (hole) density. (a) DiR with standard dimensions. (b) DiR with thicker lateral shell. The electron starts moving into the shell. (c) DiP with standard dimensions. (d) RiR with large core and giant shell. The charge separation is extremely efficient. (h) RiR with thinner core and shell. The charge separation is suppressed.

core to dilate, relaxing the strain in orthogonal directions. Consequently, the strain near the $\mathrm{CdSe} / \mathrm{CdS}$ interfaces along the $c$ axis, which is chiefly responsible for $P_{z}$, is reduced, see for example, Figure 4 in ref 41 . The overall result is that PZ effects in DiRs are weak. This is precisely what we see in Figure 5a, where we plot the exciton electron and hole charge densities in a DiR: the carriers are well confined inside the core in spite of the relatively large size we have assumed $(R=2 \mathrm{~nm})$. We note, however, that the picture would change if thicker shells could be grown, increasing the strain of the system. As shown in Figure 5b, keeping the same rod length as before but increasing $H_{\perp}$, the electron already starts moving toward the CdS shell, reducing the electron-hole overlap. We note that the enhancement of PZ fields with radial shell thickness explains recent experiments of Coropceanu et al., ${ }^{42}$ where the exciton lifetime of DiRs steadily increased from 20 to 60 ns with increasing shell thickness. For very thick shells, we predict a complete migration of the electron into the shell (Figure S8a in SI), so even longer lifetimes should be expected.

DiPs differ from DiRs mainly in that the shell is nearly twodimensional, with the $c$ axis pointing along the strong confinement direction. ${ }^{26}$ Yet, their behavior in terms of PZinduced electron delocalization (Figure 5c) is partly analogous to that of DiR: the thin shells along the $c$ axis weaken the strain. This in turn leads to weak PZ effects and carriers localized inside the core. As in the case of DiRs, electron-hole distance could be readily increased if thicker shells were grown (Figure $\mathrm{S} 8 \mathrm{~b})$.

The most favorable condition to maximize PZ effects is however achieved in recently synthesized RiRs. ${ }^{28}$ The giant shell grants strong strain. The prolate shape makes strain highly anisotropic. The long core allows for huge dipole moments, and longitudinal quantum confinement is too weak to compete. The result, as plotted in Figure $5 \mathrm{~d}$, is that electron and hole are separated toward opposite $\mathrm{CdSe} / \mathrm{CdS}$ interfaces along the $c$ axis, which explains the record exciton lifetimes measured in these structures. ${ }^{28}$ It is worth stressing that the giant shell plays a decisive role in RiRs too. RiRs with smaller core and shell width were previously synthesized by Sitt and co-workers, but long exciton lifetimes were not reported in such a case. ${ }^{27} \mathrm{We}$ simulate RiRs with similar dimensions to theirs in Figure 5e. As can be seen, in spite of the high aspect ratio and the weak longitudinal confinement, charge separation is completely suppressed, with both electron and hole localizing inside the core. The underlying reason is again the weaker strain of the system, which results in a PZ field unable to compete against electron-hole Coulomb interaction.

An important conclusion from Figure 5 is that $\mathrm{PZ}$ successfully rationalizes the very different exciton lifetimes reported for different kinds of WZ CdSe/CdS NCs. In RiRs, ${ }^{28}$ lifetimes can be 1 order of magnitude longer than those in giant-shell DiDs (ref 18 and Figure 4), and these in turn are about 1 order of magnitude longer than those in DiRs. ${ }^{24}$ This result cannot be interpreted in terms of quantum confinement because all structures have voluminous shells, but it is perfectly consistent with the different degrees of PZ-induced electronhole separation we calculate.

To summarize, we have elucidated the conditions where strain-induced piezoelectricity becomes a practical mechanism for electron-hole spatial separation in several kinds of WZ $\mathrm{CdSe} / \mathrm{CdS}$ NCs. The PZ field requires anisotropic strain, which is present even in spherical heterostructures due to the anistropy of the WZ lattice. PZ charges thus accumulate on the $\mathrm{CdSe} / \mathrm{CdS}$ interfaces, forming a dipole and a sizable built-in field along the $c$ axis, which enables directional charge separation. The magnitude of the PZ field can be enhanced using thick shells all around the core, which increase the strain in and around it, and using prolate cores, which reinforce the inherent strain anisotropy. The influence of $\mathrm{PZ}$ potential on the exciton wave function scales with the core size, as so does the PZ dipole moment, and on the shell thickness, as it provides space for the electron to escape from the core.

We have then shown that significant PZ effects are present not only in RiRs, as recently reported in ref 28 but also in giantshell DiDs and, to a lesser extent, in DiRs. In fact, the different strength of PZ fields in each kind of structure interprets the large variations of radiative lifetime reported in the literature. The present results show that, with appropiate design, band alignment can be engineered all the way from type-I or quasitype-II to a fully type-II one in any kind of CdSe/CdS NC, thus providing an efficient tool for tailoring electron-hole separation. 


\section{ASSOCIATED CONTENT}

\section{S Supporting Information}

The Supporting Information is available free of charge on the ACS Publications website at DOI: 10.1021/acs.jpclett.6b00622.

Details of methods, supporting calculations of strain maps, band-edge structure, wave functions, electronhole overlap under different conditions, a list of the material parameters employed and further experimental data. (PDF)

\section{AUTHOR INFORMATION}

\section{Corresponding Author}

*E-mail: josep.planelles@uji.es.

\section{Notes}

The authors declare no competing financial interest.

\section{ACKNOWLEDGMENTS}

Support from MINECO project CTQ2014-60178-P, UJI project P1-1B2014-24 and a FPU grant (C.S.) is acknowledged. The present publication is further realized with the support of the Ministero degli Affari Esteri e della Cooperazione Internazionale (IONX-NC4SOL, I.M.).

\section{REFERENCES}

(1) Delerue, C.; Lannoo, M. Nanostructures: Theory and Modelling; Springer: Berlin, 2004.

(2) Garcia-Santamaria, F.; Chen, Y.; Vela, J.; Schaller, R. D.; Hollingsworth, J. A.; Klimov, V. I. Suppressed Auger Recombination in "Giant" Nanocrystals Boosts Optical Gain Performance. Nano Lett. 2009, 9, 3482-3488.

(3) Cragg, G. E.; Efros, A. L. Suppression of Auger Processes in Confined Structures. Nano Lett. 2010, 10, 313-317.

(4) Climente, J. I.; Movilla, J. L.; Planelles, J. Auger Recombination Suppression in Nanocrystals with Asymmetric Electron-Hole Confinement. Small 2012, 8, 754-759.

(5) Brovelli, S.; Schaller, R. D.; Crooker, S. A.; Garcia-Santamaria, F.; Chen, Y.; Viswanatha, R.; Hollingsworth, J. A.; Htoon, H.; Klimov, V. I. Nano-engineered Electronhole Exchange Interaction Controls Exciton Dynamics in CoreShell Semiconductor Nanocrystals. Nat. Commun. 2011, 2, 280.

(6) McDaniel, H.; Pelton, M.; Oh, N.; Shim, M. Effects of Lattice Strain and Band Offset on Electron Transfer Rates in Type-II Nanorod Heterostructures. J. Phys. Chem. Lett. 2012, 3, 1094-1098.

(7) Kovalenko, M.; Manna, L.; Cabot, A.; Hens, Z.; Talapin, D. V.; Kagan, Ch. R.; Klimov, V. I.; Rogach, A. L.; Reiss, P.; Milliron, D. J.; et al. Prospects of Nanoscience with Nanocrystals. ACS Nano 2015, 9, 1012-1057.

(8) Smith, A. M.; Mohs, A. M.; Nie, S. Tuning the Optical and Electronic Properties of Colloidal Nanocrystals by Lattice Strain. Nat. Nanotechnol. 2009, 4, 56-63.

(9) Yang, S.; Prendergast, D.; Neaton, J. B. Strain-Induced Band Gap Modification in Coherent Core/Shell Nanostructures. Nano Lett. 2010, 10, 3156-3162.

(10) Fairclough, S. M.; Tyrrell, E. J.; Graham, D. M.; Lunt, P. J. B.; Hardman, S. J. O.; Pietzsch, A.; Hennies, F.; Moghal, J.; Flavell, W. R.; Watt, A. A. R.; Smith, J. M. Growth and Characterization of Strained and Alloyed Type-II ZnTe/ZnSe Core-Shell Nanocrystals. J. Phys. Chem. C 2012, 116, 26898-26907.

(11) Yadav, S. K.; Sharma, V.; Ramprasad, R. Controlling Electronic Structure Through Epitaxial Strain in $\mathrm{ZnSe} / \mathrm{ZnTe}$ Nano-Heterostructures. J. Appl. Phys. 2015, 118, 015701.

(12) Khoo, K. H.; Arantes, J. T.; Chelikowsky, J. R.; Dalpian, G. M. First-Principles Calculations of Lattice-Strained Core-Shell Nanocrystals. Phys. Rev. B: Condens. Matter Mater. Phys. 2011, 84, 075311.
(13) Phadnis, C.; Sonawane, K. G.; Hazarika, A.; Mahamuni, S. Strain-Induced Hierarchy of Energy Levels in CdS/ZnS Nanocrystals. J. Phys. Chem. C 2015, 119, 24165-24173.

(14) Pahomi, T. E.; Cheche, T. O. Strain Influence on Optical Absorption of Giant Semiconductor Colloidal Quantum Dots. Chem. Phys. Lett. 2014, 612, 33-38.

(15) Cirillo, M.; Aubert, T.; Gomes, R.; Deun, R. V.; Emplit, P.; Biermann, A.; Lange, H.; Thomsen, C.; Brainis, E.; Hens, Z. Flash” Synthesis of CdSe/CdS Core-Shell Quantum Dots. Chem. Mater. 2014, 26, 1154-1160.

(16) Park, S. H.; Cho, Y. H. Characteristics of Built-in Polarization Potentials in Vertically and Laterally Arranged InGaN/GaN Quantum Dots. J. Appl. Phys. 2011, 109, 113103.

(17) Chen, O.; Zhao, J.; Chauhan, V. P.; Cui, J.; Wong, C.; Harris, D. K.; Wei, H.; Han, H. S.; Fukumura, D.; Jain, R. K.; Bawendi, M. G. Compact High-Quality CdSe/CdS Core/Shell Nanocrystals with Narrow Emission Linewidths and Suppressed Blinking. Nat. Mater. 2013, 12, 445-451.

(18) Christodoulou, S.; Vaccaro, G.; Pinchetti, V.; De Donato, F.; Grim, J. Q.; Casu, A.; Genovese, A.; Vicidomini, G.; Diaspro, A.; Brovelli, S.; et al. Synthesis of Highly Luminescent Wurtzite CdSe/ CdS Giant-shell Nanocrystals Using a Fast Continuous Injection Route. J. Mater. Chem. C 2014, 2, 3439-3447.

(19) Mahler, B.; Lequeux, N.; Dubertret, B. Ligand-Controlled Polytypism of Thick-Shell CdSe/CdS Nanocrystals. J. Am. Chem. Soc. 2010, 132, 953-959.

(20) van Embden, J.; Jasieniak, J.; Mulvaney, P. Mapping the Optical Properties of CdSe/CdS Heterostructure Nanocrystals: The Effects of Core Size and Shell Thickness. J. Am. Chem. Soc. 2009, 131, 1429914309.

(21) Qin, H.; Niu, Y.; Meng, R.; Lin, X.; Lai, R.; Fang, W.; Peng, X. Single-Dot Spectroscopy of Zinc-Blende CdSe/CdS Core/Shell Nanocrystals: Nonblinking and Correlation with Ensemble Measurements. J. Am. Chem. Soc. 2014, 136, 179-187.

(22) Carbone, L.; Nobile, C.; De Giorgi, M.; Della Sala, F.; Morello, G.; Pompa, P.; Hytch, M.; Snoeck, E.; Fiore, A.; Franchini, I. R.; et al. Synthesis and Micrometer-Scale Assembly of Colloidal CdSe/CdS Nanorods Prepared by a Seeded Growth Approach. Nano Lett. 2007, 7, 2942-2950.

(23) Sitt, A.; Hadar, I.; Banin, U. Band-Gap Engineering, Optoelectronic Properties and Applications of Colloidal Heterostructured Semiconductor Nanorods. Nano Today 2013, 8, 494-513.

(24) Raino, G.; Stoferle, T.; Moreels, I.; Gomes, R.; Kamal, J. S.; Hens, Z.; Mahrt, R. F. Probing the Wave Function Delocalization in CdSe/CdS Dot-in-Rod Nanocrystals by Time- and TemperatureResolved Spectroscopy. ACS Nano 2011, 5, 4031-4036.

(25) Wu, K.; Rodriguez-Cordoba, W. E.; Liu, Z.; Zhu, H.; Lian, T. Beyond Band Alignment: Hole Localization Driven Formation of Three Spatially Separated Long-Lived Exciton States in CdSe/CdS Nanorods. ACS Nano 2013, 7, 7173-7185.

(26) Cassette, E.; Mahler, B.; Guigner, J. M.; Patriarche, G.; Dubertret, B.; Pons, T. Colloidal CdSe/CdS Dot-in-Plate Nanocrystals with 2D-Polarized Emission. ACS Nano 2012, 6, 6741-6750.

(27) Sitt, A.; Salant, A.; Menagen, G.; Banin, U. Highly Emissive Nano Rod-in-Rod Heterostructures with Strong Linear Polarization. Nano Lett. 2011, 11, 2054-2060.

(28) Christodoulou, S.; Rajadell, F.; Casu, A.; Vaccaro, G.; Grim, J.; Genovese, A.; Manna, L.; Climente, J. I.; Meinardi, F.; Raino, G.; et al. Band Structure Engineering via Piezoelectric Fields in Strained Anisotropic CdSe/CdS Nanocrystals. Nat. Commun. 2015, 6, 7905.

(29) Talapin, D. V.; Nelson, J. H.; Shevchenko, E. V.; Aloni, S.; Sadtler, B.; Alivisatos, A. P. Seeded Growth of Highly Luminescent CdSe/CdS Nanoheterostructures with Rod and Tetrapod Morphologies. Nano Lett. 2007, 7, 2951-2959.

(30) Fiore, A.; Mastria, R.; Lupo, M. G.; Lanzani, G.; Giannini, C.; Carlino, E.; Morello, G.; De Giorgi, M.; Li, Y.; Cingolani, R.; Manna, L. Tetrapod-Shaped Colloidal Nanocrystals of II-VI Semiconductors Prepared by Seeded Growth. J. Am. Chem. Soc. 2009, 131, 2274-2282. 
(31) Deka, S.; Miszta, K.; Dorfs, D.; Genovese, A.; Bertoni, G.; Manna, L. Octapod-Shaped Colloidal Nanocrystals of Cadmium Chalcogenides via "One-Pot" Cation Exchange and Seeded Growth. Nano Lett. 2010, 10, 3770-3776.

(32) Eshet, H.; Grünwald, M.; Rabani, E. The Electronic Structure of CdSe/CdS Core/Shell Seeded Nanorods: Type-I or Quasi-Type-II? Nano Lett. 2013, 13, 5880-5885.

(33) Shabaev, A.; Rodina, A. V.; Efros, A. L. Fine Structure of the Band-Edge Excitons and Trions in CdSe/CdS Core/Shell Nanocrystals. Phys. Rev. B: Condens. Matter Mater. Phys. 2012, 86, 205311.

(34) Langbein, W.; Hetterich, M.; Grun, M.; Klingshirn, C.; Kalt, H. Picosecond Luminescence Dynamics in CdS/CdSe Stark Superlattices. Appl. Phys. Lett. 1994, 65, 2466-2468.

(35) Andreev, A. D.; O'Reilly, E. P. Theory of the Electronic Structure of GaN/AlN Hexagonal Quantum Dots. Phys. Rev. B: Condens. Matter Mater. Phys. 2000, 62, 15851-15870.

(36) Seguin, R.; Schliwa, A.; Rodt, S.; Potschke, K.; Pohl, U. W.; Bimberg, D. Size-dependent Fine-Structure Splitting in Self-Organized InAs/GaAs Quantum Dots. Phys. Rev. Lett. 2005, 95, 257402.

(37) Morello, G.; Della Sala, F.; Carbone, L.; Manna, L.; Maruccio, G.; Cingolani, R.; De Giorgi, M. Intrinsic Optical Nonlinearity in Colloidal Seeded Grown CdSe/CdS Nanostructures: Photoinduced Screening of the Internal Electric Field. Phys. Rev. B: Condens. Matter Mater. Phys. 2008, 78, 195313.

(38) Zhu, B. H.; Zhang, H. C.; Zhang, Z. Y.; Cui, Y. P.; Zhang, J. Y. Effect of Shell Thickness on Two-Photon Absorption and Refraction of Colloidal CdSe/CdS Core/Shell Nanocrystals. Appl. Phys. Lett. 2011, 99, 231903.

(39) Luo, Y.; Wang, L. W. Electronic Structures of the CdSe/CdS Core-Shell Nanorods. ACS Nano 2010, 4, 91-98.

(40) Muller, J.; Lupton, J. M.; Lagoudakis, P. G.; Schindler, F.; Koeppe, R.; Rogach, A. L.; Feldmann, J.; Talapin, D. V.; Weller, H. Wave Function Engineering in Elongated Semiconductor Nanocrystals with Heterogeneous Carrier Confinement. Nano Lett. 2005, 5, 20442049.

(41) Rajadell, F.; Royo, M.; Planelles, J. Strain in Free Standing CdSe/CdS Core-Shell Nanorods. J. Appl. Phys. 2012, 111, 014303.

(42) Coropceanu, I.; Rossinelli, A.; Caram, J. R.; Freyria, F. S.; Bawendi, M. G. Slow-Injection Growth of Seeded CdSe/CdS Nanorods with Unity Fluorescence Quantum Yield and Complete Shell to Core Energy Transfer. ACS Nano 2016, 10, 3295-3301. 